Voix et Images

volxetimages

\title{
Du comment-lire au commentaire : lecture(s) féministes(s)
}

\section{Danielle Fournier}

Volume 15, numéro 1 (43), automne 1989

Jacques Poulin

URI : https://id.erudit.org/iderudit/200824ar

DOI : https://doi.org/10.7202/200824ar

Aller au sommaire du numéro

\section{Éditeur(s)}

Université du Québec à Montréal

\section{ISSN}

0318-9201 (imprimé)

1705-933X (numérique)

Découvrir la revue

\section{Citer cet article}

Fournier, D. (1989). Du comment-lire au commentaire : lecture(s) féministes(s).

Voix et Images, 15(1), 124-127. https://doi.org/10.7202/200824ar d'utilisation que vous pouvez consulter en ligne.

https://apropos.erudit.org/fr/usagers/politique-dutilisation/ 
124 VOIX \& IMAGES / 43, automne 1989

\title{
Du comment-lire au commentaire: lecture(s) féministe(s)
}

\author{
par Danielle Fournier, université McGill
}

Les livres qui veulent en même temps être analytiques - au sens littéraire du terme - et théoriques posent toujours cette double difficulté: comment rendre justice aux deux parties, aussi importantes l'une que l'autre? L'essai de 
Patricia Smart, Écrire dans la maison du père ${ }^{1}$, publié chez Québec/ Amérique, n'y échappe pas. La partie consacrée à l'analyse des œuvres littéraires est pertinente et particulièrement utile pour quiconque travaille le texte; la réflexion ou les a priori de la réflexion, tant dans l'introduction que dans ce qui sous-tend les analyses, restent didactiques et pédagogiques. Le texte séminal de Laure Conan, Angéline de Montbrun, contribue à donner cette idée de linéarité à l'essai.

Cet essai aura été capital en 1989: d'une part, il a été couronné par le prix du Gouverneur général, d'autre part, il continue une certaine réflexion déjà amorcée dans le champ culturel et littéraire par le féminisme. La lecture de Patricia Smart se donne comme cet autre regard posé sur le monde, celui d'un féminisme qui questionne le personnage comme personnage social.

Ainsi cet essai tente de quitter la voie du lecteur universel et neutre pour suivre celle d'une lectrice engagée dans son processus de lecture: le lire-femme. Première constatation: que des femmes mortes ou tuées. Pour Smart, commencer à lire en tant que femme (p. 20) déplace l'angle de vision et la voix qui se fait entendre devient subversive. L'objet femme, quand il se met à écrire, commence à se percevoir comme un sujet (p. 23) et accède à l'autorité.

La lecture est une sorte d'itinéraire; le point de vue pédagogique et l'enseignement universitaire occupent une place importante: on ne lit pas des notes de cours, mais derrière une écriture adroite, il y a cet objectif de dire le non-dit et le non-entendu d'une littérature soumise à une «volonté» patriarcale. Pour Smart, il s'agit de la contradiction principale du Québec: cette attitude devant le féminin.

Ses analyses - que ce soit celles des romans du terroir, de l'œuvre d'Hubert Aquin, de Gabrielle Roy ou de Germaine Guèvremont, pour ne nommer que quelques auteurs - sont toujours mises en rapport avec le roman de Laure Conan, Angéline de Montbrun. Du strict point de vue pédagogique, ces analyses contribuent à penser les œuvres littéraires comme paradigmatiques, mais surtout, comme porteuses d'un féminin refoulé. Toutes ces études, depuis le roman de Laure Conan, rendent compte des rapports inter-personnages comme sujets potientellement sociaux avec l'avènement du féminisme.

Le travail de l'essayiste permet certes d'amorcer de nouvelles discussions - les idées sont là pour être pensées - et entre autres celles sur le statut autobiographique du «je» féminin dans/de l'écriture. Ce qu'elle tisse autour des écritures québécoises donne envie de lire les textes et de confronter cette réflexion à d'autres positions autrement féministes. Mais il y a plus. Il y a débordement de sens: nul besoin de rappeler que le vouloir-dire est toujours largement dépassé par le dire lui-même et qu'il est associé à la différence sexuelle. Il sera donc question d'articuler à cette différence le paradoxe de la modernité puisque la modernité irait de concert avec la différence sexuelle.

Cependant, reste marqué dans la pensée féministe ce désir de la «dichotomisation» de la différence sexuelle. Cette analyse du féminin réduit la représentation à du «un», de l'univoque. 
Il semble que la différence sexuelle, chez Patricia Smart, n'est pas parlante à partir du sexe comme toujours manquant, comme manquant de toute façon, mais qu'elle se pose en deux termes opposés et surtout contradictoires, en deux lieux et en deux objets: l'homme et la femme. Or, ici le féminin demeurant le contraire du masculin, le lieu d'exercice de sa parole reste seul soumis aux lois de et dans la maison du Père parce que c'est là et uniquement là que la parole peut devenir langage. Son introduction, «Les traces d'un meurtre», va dans le sens des hypothèses actuelles de recherches féministes.

Parfois, cependant, on sent que le cliché n'est pas loin. Cette idée dualiste du bon féminin et du mauvais masculin en regard de leur rapport à la violence - la violence se fait toujours contre les femmes - peut agacer. Elle semble, en tout cas, datée historiquement. Peut-être pose-t-elle autrement la question du féminisme: comment peut-il traverser l'histoire sans pour autant limiter les points de vue?

Il n'en demeure pas moins que la connaissance des œuvres reste entière. La perspective, d'abord historique et chronologique, oriente la lecture en regard du passage du texte d'écriture au texte littéraire. Les romans sont des romans déjà reçus, comme on dit, reçus et par l'institution et par la culture.

On ne peut lire autrement que depuis son sexe, son origine de classe, etc. On ne peut pas non plus lire en dehors de son époque et de son histoire personnelle et collective; l'équivalent me semble vrai pour l'écriture: on ne peut écrire en dehors du monde qui nous entoure. À cet égard, et comme le suggère Patricia Smart, les cuvres d'art explorent la contradiction. Reste à savoir - si ce savoir se peut - laquelle contradiction!... Mais son livre, et voilà son mérite, est un livre ouvert et qui ouvre l'horizon des recherches contemporaines.

Les écritures de femmes sont fragmentaires: est-ce là l'apanage des femmes? On voudrait bien le croire. L'écriture reste fragmentée, la littérature, plus particulièrement les études littéraires, s'occupent de coller ensemble les voix, les pièces disparates, les mots décousus, les récits sans histoire, etc. Si on ne conteste pas la réelle difficulté qu'ont les femmes dans leur venue à l'écriture, il n'en demeure pas moins que dans le geste d'écriture, que l'on soit homme ou femme, le sujet est seul au monde, avec sa violence, sa passion, son sexe, son désir et son amour. À trop vouloir rendre sujet social le sujet écrivant, Patricia Smart déplace peut-être, au profit du réalisme, ce qui s'en était échappé.

Cet essai laisse entendre, comme d'autres essais dits féministes, que l'écriture est plus facile pour les hommes que pour les femmes. Que la position mâle, sans par ailleurs être enviable, demeure une position d'autorité: de cette autorité naît la maison. De la maison, la violence. La conclusion du livre donne toutefois à penser, je ne sais ni pourquoi ni comment, que les nouvelles écritures ouvrent un autre espace malgré la difficulté qu'éprouvent les femmes à s'insérer dans le registre du culturel. Je crois qu'il y a là confusion au niveau du sens: le symbolique, c'est le langage auquel on est tous soumis, parfois à notre corps défendant. 
Écrire, c'est chaque fois se demander non seulement son sexe, mais aussi d'où on vient et qui on est, où on va et de qui on vient. J'ajouterais que le sujet social, au sens du lieu social comme lien, ne choisit pas l'écriture de fiction: il peut écrire, homme ou femme, mais il n'est pas évident que sa production en soit une de fiction. Écrire de la fiction, c'est être décidé par elle, dans la violence que reproche Patricia Smart aux hommes, et dans la douceur, si la douceur est le contraire de la violence. Être décidé par la fiction détermine le sujet en ce qu'il n'est pas dans la maîtrise de lui-même et encore dans la maîtrise de son texte. Peut-être la psychanalyse laisse-t-elle cette plage du texte iouverte.

Cependant, le principal reproche qui doit être fait à ce livre, non pas à l'auteur mais bien à l'éditeur, c'est qu'il n'y a ni bibliographie ni index analytique. Comment un livre qui est un outil de travail pédagogique et de réflexion théorique peut-il ne pas avoir ces références minimales?

Pour terminer, cet ouvrage trouvera sa place et contentera son auditoire. Patricia Smart propose son point de vue, mais les pensées multiples, singulières et différentes se développent. S'il y a émergence du féminin, et il semble bien que ce soit le cas, ce ne peut être que lié au féminin lui-même en ce sens qu'il est une pensée du féminin. Aussi importante que soit devenue cette question elle sera sans doute la pensée de ce siècle —, aussi importante doit être la possibilité de tenir un discours de vie qui ne tue pas et qui ne ramène pas tout à des présupposés idéologiques qui, au lieu d'ouvrir les discours, les ferment.

1 Patricia Smart, Écrire dans la maison du père. L'émergence du féminin dans la tradition littéraire du Québec, essai, Montréal, Québec/Amérique, 1988, 340 p. (Littérature d'Amérique). 